\title{
Intratumoral Injection of ${ }^{188}$ Re labeled Cationic Polyethylenimine Conjugates: A Preliminary Report
}

\begin{abstract}
${ }^{188} \mathrm{Re}$ (Rhenium) is easily obtained from an in-house ${ }^{188} \mathrm{~W} /{ }^{188} \mathrm{Re}$ generator that is similar to the current ${ }^{99} \mathrm{Mo} /{ }^{19 m} \mathrm{Tc}$ generator, making it very convenient for clinical use. This characteristic makes this radionuclide a promising candidate as a therapeutic agent. Polyethylenimine (PEI) is a cationic polymer and has been used as a gene delivery vector. Positively charged materials interact with cellular blood components, vascular endothelium, and plasma proteins. In this study, the authors investigated whether intratumoral injection of ${ }^{188} \mathrm{Re}$ labeled transferrin (Tf)-PEI conjugates exert the effect of radionuclide therapy against the tumor cells. When the diameters of the Ramos lymphoma (human Burkitt's lymphoma) xenografted tumors reached approximately $1 \mathrm{~cm}, 3$ kinds of ${ }^{188} \mathrm{Re}$ bound compounds (HYNIC-PEI-Tf, HYNIC-PEI, ${ }^{188}$ Re perrhenate) were injected directly into the tumors. There were increases in the retention of ${ }^{188} \mathrm{Re}$ inside the tumor when PEI was incorporated with ${ }^{188} \mathrm{Re}$ compared to the use of free ${ }^{188} \mathrm{Re}$. The ${ }^{188} \mathrm{Re}$ HYNIC-Tf-PEI showed the most retention inside the tumor (retention rate=approximately $97 \%$ ). H\&E stain of isolated tumor tissues showed that ${ }^{188} \mathrm{Re}$ labeled HYNIC-PEI-Tf caused extensive tumor necrosis. These results support ${ }^{188} \mathrm{Re}$ HYNIC-PEI-Tf as being a useful radiopharmaceutical agent to treat tumors when delievered by intratumoral injection.
\end{abstract}

Key Words : Rhenium; Polyethylenimine; Transferrin; Lymphoma

\author{
Eun-Mi Kim ${ }^{\star}$, Hwan-Jeong Jeong ${ }^{*,}$, \\ Young-Jun $\mathrm{Heo}^{\S}$, Hyung-Bae Moon ${ }^{\dagger \neq}$, \\ Hee-Seung Bom ${ }^{\S}$, Chang-Guhn Kim \\ *Department of Nuclear Medicine; 'Wonkwang \\ University Institute of Medical Science; 'Department of \\ Pathology, Wonkwang University School of Medicine, \\ Iksan; ${ }^{\circledR}$ Department of Nuclear Medicine, Chonnam \\ National University School of Medicine, Gwangju, \\ Korea
}

Received : 15 March 2004

Accepted : 20 May 2004

Address for correspondence

Hwan-Jeong Jeong. M.D.

Department of Nuclear Medicine, Wonkwang University Hospital, 344-2 Sinyong-dong, Iksan 570-711, Korea

Tel : +82.63-850-1367. Fax: +82.63-852-1310

E-mail : jayjeong@wonkwang.ac.kr

\section{INTRODUCTION}

${ }^{188} \mathrm{Re}$ (Rhenium) is a short-lived beta emitting radionuclide (physical half-life $=17 \mathrm{hr}, E \max =2.11 \mathrm{MeV}$ ). It has an average beta particle penetration of $3.3 \mathrm{~mm}$ (maximum $=10.8)$, providing a tightly circumscribed region of high-energy deposition with little damage to the adjacent cells and organs. In addition, ${ }^{188} \mathrm{Re}$ is easily obtained from an in-house ${ }^{188} \mathrm{~W} /{ }^{188} \mathrm{Re}$ generator similar to the current ${ }^{99} \mathrm{Mo} /{ }^{99} \mathrm{~m}$ Tc generator, which makes it convenient for clinical use (1-4). These characteristics make this radionuclide a promising candidate as a therapeutic agent.

Polyethylenimine (PEI) is a cationic polymer that has recently appeared as a possible alternative to viral and liposomal routes for gene delivery (5). PEI has been studied as a chelating agent to remove heavy metal ions $(6,7)$. Because PEI contains primary, secondary, and tertiary amines, PEI derivatives can be chelated with a radionuclide such as ${ }^{188}$ Re. PEI can also be coupled to ligands such as galactose or transferrin (Tf) for the purpose of targeting specific organ or tumor cells (8-12). Therefore, ligand binding PEI derivatives labeled with ${ }^{188} \mathrm{Re}$ may be used as a novel radiopharmaceutical in targeting therapy for tumor treatment.

Use of Tf conjugates for site-specific drug delivery and gene delivery to tumor cells has been studied via intravenous injec- tion $(13,14)$. Xu et al. reported that an intratumoral injection using the Tf-liposome system showed a higher number of transfected tumor cells in vivo when compared with transfection by liposome alone (15). These results indicate that irrespective of the route of injection, Tf conjugates specifically bind to Tf receptors (Tf-R) present in the tumor cell membrane. These complexes then enter the cells by receptor-mediated endocytosis. This study investigated whether intratumoral injections of ${ }^{188} \mathrm{Re}$ labeled Tf-PEI conjugates exert the full effect of radionuclide therapy against the tumor cells.

\section{MATERIALS AND METHODS}

\section{Synthesis of HYNIC-PEI-Tf conjugates}

Tf-PEI (branched PEI, $25 \mathrm{kDa}$ ) conjugates were synthesized as described by Kircheis and coworkers (16). Branched PEI was purchased from Aldrich (WI, U.S.A.). Briefly, human apo-transferrin in $30 \mathrm{mM}$ sodium acetate buffer ( $\mathrm{pH}$ 5.0) was subjected to gel filtration on a Sephadex G-25 superfine column (Pharmacia, Uppsala, Sweden). The resulting solution was cooled to $0^{\circ} \mathrm{C}$ and sodium periodate (in $30 \mathrm{mM} \mathrm{pH} 5$ sodium acetate buffer) were added. The mixture was kept in an ice bath in the dark for $90 \mathrm{~min}$ and promptly added to 
PEI solution and mixed at room temperature. After $30 \mathrm{~min}$, four portions of sodium cyanoborohydride were added at 1 hr intervals. After $18 \mathrm{hr}, 3 \mathrm{M}$ sodium chloride was added. The reaction mixture was loaded on a cation-exchange column, Macro-Prep high S HR 10/10 (Bio-Rad, Hercules, CA, U.S.A.) and was fractionated with a salt gradient from 0.5 $\mathrm{M}$ to $3.0 \mathrm{M}$ sodium chloride in $20 \mathrm{mM}$ HEPES ( $\mathrm{pH} 7.3$ ). A 3 molar excess of dissolved succinimidyl 6-hydrazino nicotinate hydrochloride (HYNIC) (5 mol\% of PEI amino group) in $30 \mathrm{mM}$ dimethyl-formamide (DMF) was added dropwise to a stirred solution of Tf-PEI conjugates. The solution was stirred gently for $24 \mathrm{hr}$ at $4^{\circ} \mathrm{C}$ protected from light. This was followed by dialysis against HBS ( $\mathrm{pH} 7.3,150 \mathrm{mM}$ sodium chloride, $20 \mathrm{mM}$ HEPES) at $4{ }^{\circ} \mathrm{C}$ (six buffer changes for $72 \mathrm{hr}$ ). The iron incorporation was performed by addition of $1.25 \mu \mathrm{L}$ $10 \mathrm{mM}$ iron (III) citrate buffer per $\mathrm{mg}$ transferrin content. The conjugates were divided into convenient small aliquots and kept at $-20^{\circ} \mathrm{C}$.

\section{Labeling with ${ }^{188} \mathrm{Re}$}

${ }^{188} \mathrm{Re}$-perrhenate was obtained in $20 \mathrm{~mL}$ of normal saline from ${ }^{188} \mathrm{~W} /{ }^{188} \mathrm{Re}$ generator (Shanghai Ke Xing Pharmaceuticals, Shanghai, China). The labeling process was carried out in the presence of stannous chloride dehydrate. ${ }^{188} \mathrm{Re}$ sodium perrhenate eluate $(370 \mathrm{MBq})$ in $0.9 \%$ normal saline was mixed in a vial with $10 \mathrm{mg}$ of ascorbic acid, $40 \mathrm{mg}$ of $\mathrm{SnCl}_{2}\left(\mathrm{Si}_{-}\right.$ gma, U.S.A.), and $500 \mu \mathrm{L}$ of HYNIC-PEI-Tf $(1 \mu \mathrm{g} / \mu \mathrm{L})$ or HYNIC-PEI. Then, the vial was reacted at $37^{\circ} \mathrm{C}$ for $1 \mathrm{hr}$. The labeling yield was determined by ITLC-SG (Gelman Science, Ann Arbor, MI, U.S.A.) using acetone as the mobile phase.

\section{In vivo animal model}

To generate tumors, three 5- to 6-week-old female BALB/c nude mice (Orient Co. Ltd., Seoul, Korea) were injected subcutaneously in the left thigh with Ramos cells (ATCC CRL 1596, human Burkitt's lymphoma, $5 \times 10^{6}$ cells $/ 100 \mu \mathrm{L}$ ). Tumor size was measured using a vernier caliper across its longest and after the diameters were reached about 1 to 1.5 $\mathrm{cm}, 3$ kinds of ${ }^{188}$ Re labeled compounds $(300 \mu \mathrm{Ci}$ of HYNICPEI-Tf, HYNIC-PEI, and free ${ }^{188} \mathrm{Re}$ ) were injected directly three times into the tumors, respectively.

\section{Image acquisition}

Static images were acquired with a gamma camera (Vertex, ADAC, Milpitas, CA, U.S.A.) equipped with a low energy, pin-hole collimator, and with a $40 \%$ window centered over the $155-\mathrm{keV}$ photopeak. Gamma images were achieved from $10 \mathrm{~min}$ to $12 \mathrm{hr}$ after injection to certifying intratumoral retention of ${ }^{188} \mathrm{Re}$ HYNIC-PEI-Tf (11.1 MBq/mouse) and ${ }^{188}$ Re HYNIC-PEI in comparison with that applied with same dose of ${ }^{188} \mathrm{Re}$ perrhenate.

\section{Hematoxylin \& eosin staining}

Histologic evaluation with $\mathrm{H} \& \mathrm{E}$ staining was performed 2 days after injection. The frozen sections were washed with tap water for $5 \mathrm{~min}$, immersed in hematoxylin for $2 \mathrm{~min}$ and checked for complete staining in tap water. Eosin staining was carried out for $3 \mathrm{~min}$. Sections were dehydrated through a graded series of alcohol (70 to $100 \%$ ethanol, 3 min each), cleared in xylene, cover-slipped and observed with a light microscope.

\section{Image analysis and dosimetry}

Region of interest (ROI) were drawn manually on $10 \mathrm{~min}$ image around the tumor and whole body. ROIs of the same size and shape were applied to $2 \mathrm{hr}$ and $12 \mathrm{hr}$ images. Retention rates of 3 radiotracers were calculated. A half-life for ${ }^{188} \mathrm{Re}$ HYNIC-PEI-Tf was also calculated in the form of effective half-life and the residence time was defined as half-life/ln 2. Tumor self-radiation S-value of ${ }^{188} \mathrm{Re}$ HYNIC-PEI-Tf was obtained using the Nodule Module in MIRDOSE3.1 software (Oak Ridge Associated University) (17). From the calculated S-values and residence time in the mouse model, the dose of ${ }^{188}$ Re HYNIC-PEI-Tf to obtain 100 Gy of tumor irradiation was calculated.

\section{Reverse transcription-polymerase chain reaction}

Total RNA isolated from Ramos cells with Trizol reagent (Invitrogen, U.S.A.) was used for RT-PCR. First-strand cDNA synthesis was performed at $42^{\circ} \mathrm{C}$ for $50 \mathrm{~min}$, followed by 70 ${ }^{\circ} \mathrm{C}$ for 15 min using Superscript II reverse transcriptase and oligo $(\mathrm{dT})_{10}$ as the primer. The cDNA was amplified using the following primers: Tf-R F 5' CTCACTTTAGACAATGCTGC 3'; Tf-R R 5' CTCATGACACGATCATTGAG 3'. A pair of primers specific to $\beta$-actin (F $5^{\prime}$-TGACGGGGTCACCCACACTGTGCCCATC TA-3'; R 5'-CTAGAAGCATTTGCGGTGGACGATGCAGGG-3') was used as a control. PCR was performed in a DNA thermal cycler using $94^{\circ} \mathrm{C}$ melting, $45^{\circ} \mathrm{C}$ annealing, and $72^{\circ} \mathrm{C}$ extension temperatures for 33 cycles. The PCR products were loaded on agarose gel $(1 \%)$ containing ethidiume bromide and electrophoresis was performed at $100 \mathrm{~V}$ for $20 \mathrm{~min}$.

\section{RESULTS}

The labeled ${ }^{188}$ Re HYNIC-PEI-Tf or HYNIC-PEI remained localized at the origin of injection and radiochemical purities of these labeled compounds at $15 \mathrm{~min}$ and $1 \mathrm{hr}$ were $97 \%$ and $80 \%$, respectively.

Fig. 1A, B showed that when PEI was incorporated with ${ }^{188} \mathrm{Re}$, the retention of ${ }^{188} \mathrm{Re}$ inside the tumor was increased compared with free ${ }^{188}$ Re. The ${ }^{188}$ Re HYNIC-PEI-Tf mostly 


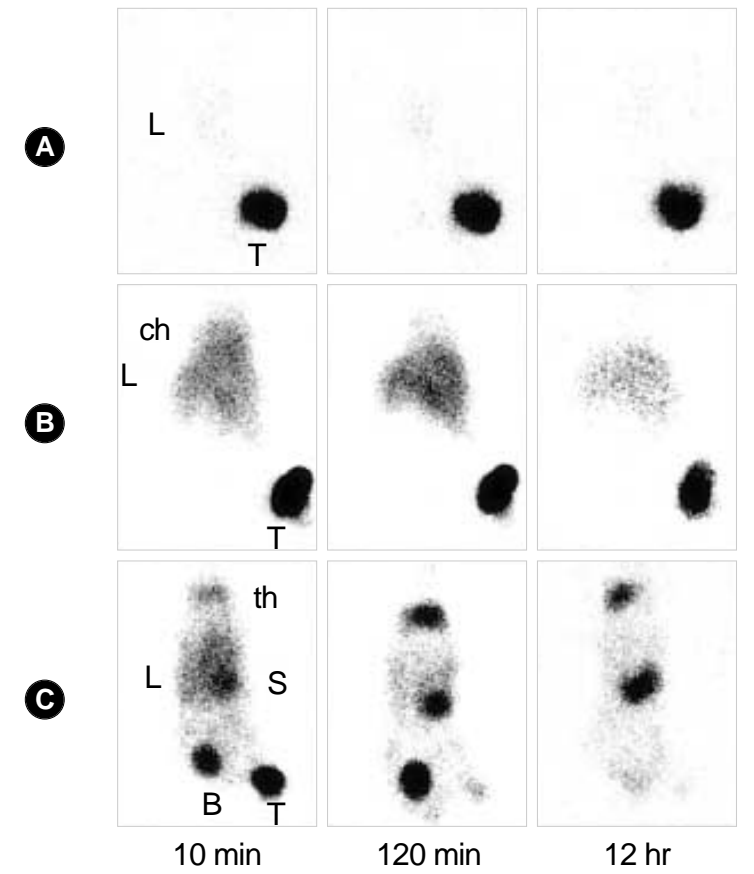

Fig. 1. Gamma images of Ramos lymphoma xenografted nude mice $10 \mathrm{~min}, 2 \mathrm{hr}$, and $12 \mathrm{hr}$ after intratumoral injection. (A) Of three kinds of ${ }^{188}$ Re conjugates, ${ }^{188}$ Re HYNIC-PEI-Tf showed the highest retention rate inside the tumor and relatively no leakage from the tumor when radionuclides were injected intratumorally. (B) With time, ${ }^{188} \mathrm{Re}$ HYNIC-PEl escaped from the tumor with some extent (approximately 15\%) and accumulated into the lung and liver.

(A) ${ }^{188}$ Re HYNIC-PEI-Tf injected mouse, (B) ${ }^{188}$ Re HYNIC-PEl injected mouse, $(C){ }^{188}$ Re perrhenate injected mouse.

L, liver; T, tumor; ch, chest; th, thyroid; S, stomach; B, bladder.

remained in the tumor and showed a higher retention rate than ${ }^{188}$ Re HYNIC-PEI (approximately $97 \%$ vs. 85\%). ${ }^{188} \mathrm{Re}$ HYNIC-PEI released from the tumor accumulated in the liver and lungs. Fig. $1 \mathrm{C}$ showed that free ${ }^{188} \mathrm{Re}$ escaped from the tumor over time and that there was no remaining ${ }^{188} \mathrm{Re}$ $12 \mathrm{hr}$ after injection. The ${ }^{188} \mathrm{Re}$ released accumulated in the stomach, thyroid, and bladder in much the same way that ${ }^{99 \mathrm{~m}}$ Tc pertechnetate did.

Effective half-life of ${ }^{188}$ Re HYNIC-PEI-Tf was assumed to be same to the physical half-life. The caculated residence time of this conjugate was $24.5 \mathrm{hr}$. The nodular self-dose S-value was estimated as $0.343 \mathrm{mGy} / \mathrm{MBq} \cdot \mathrm{h}$. using MIRDOSE3.1. The radioactivity required for a target irradiation dose of 100 Gy for 0.97 to $1.23 \mathrm{~cm}$ tumor was calculated 6.4 to $11.9 \mathrm{MBq}$ for ${ }^{188}$ Re HYNIC-PEI-Tf from this S-value.

Representative hematoxylin and eosin-stained sections of the isolated tumors injected with ${ }^{188}$ Re HYNIC-PEI-Tf and ${ }^{188} \mathrm{Re}$ perrhenate were shown in Fig. 2. In the tumor injected with ${ }^{188} \mathrm{Re}$ HYNIC-PEI-Tf, histological changes were found in contrast with ${ }^{188}$ Re perrhenate. ${ }^{188}$ Re HYNIC-PEI-Tf made extensive central necrosis inside the tumor and remained the small portion of viable tumor tissue around the tumor mass.

The result of RT-PCR represented the Ramos Burkitt's

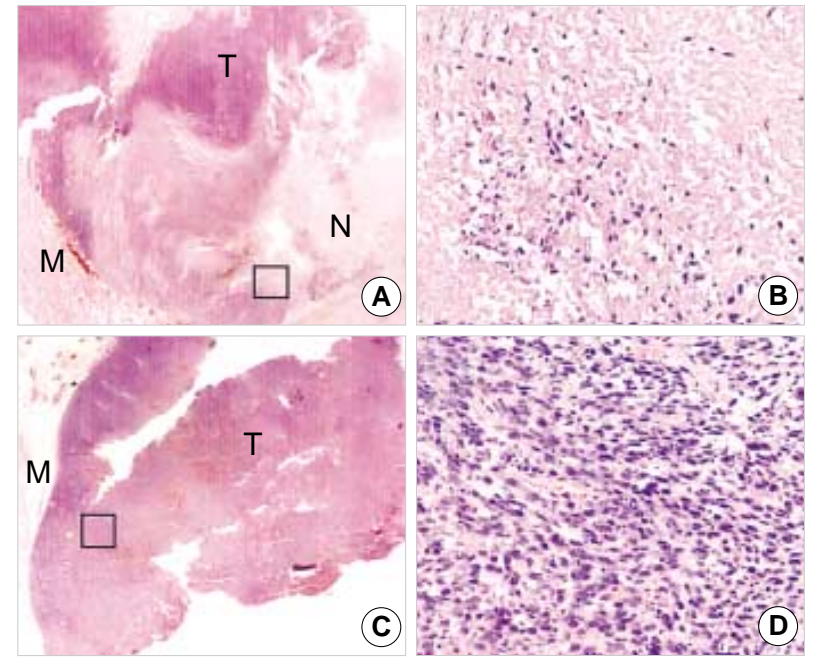

Fig. 2. Hematoxylin-eosin staining obtained $48 \mathrm{hr}$ after intratumoral injection. (A, B) Histological findings (H\&E, original magnification $\times 10, \times 200$ ) demonstrate that wide central necrosis with peripheral viable cells is shown when ${ }^{188}$ Re HYNIC-PEI-Tf is injected, but $(C, D, H \& E \times 10, \times 200)$ no significant necrosis when ${ }^{188}$ Re perrhenate injected.

T, tumor; N, necrosis; M, muscle.

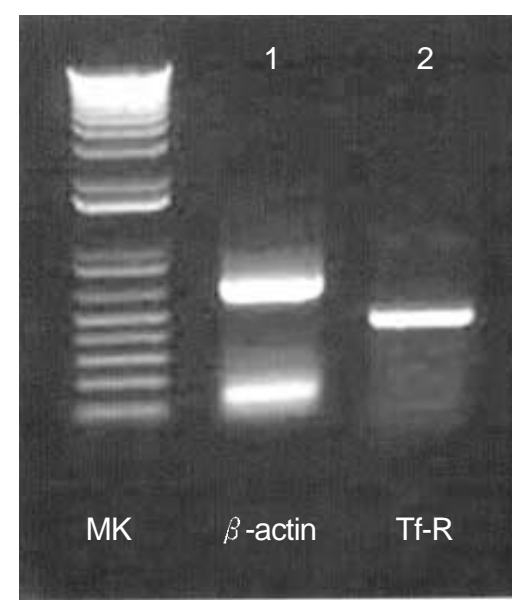

Fig. 3. The result of RT-PCR for Tf-receptor in Ramos lymphoma cells. The result demonstrates that Ramos lymphoma cells had mRNA of human Tf-receptor. Lane 1: $\beta$ actin as control, Lane 2: Tf-R. MK: Molecular size marker.

lymphoma cells had Tf-R mRNA and the possibility of expression of Tf-R protein on the cell membrane (Fig. 3).

\section{DISCUSSION}

Our preliminary results show that intratumoral injection with ${ }^{188}$ Re HYNIC-PEI-Tf caused extensive necrosis in the xenografted tumor without a significant leakage of radioactive compound. Although PEI could chelate with ${ }^{99 \mathrm{~m}} \mathrm{Tc}$ or ${ }^{188} \mathrm{Re}$ because of the secondary or tertiary amine groups in this polymer, in this study HYNIC was introduced as a biconjugate for higher and safer labeling.

${ }^{188}$ Re has not only strong potential for therapeutic use but also excellent imaging characteristics because of its $\beta$ energy 
(2.1 MeV), its short physical half-life (17 hr) and its $155 \mathrm{keV}$ $\gamma$-ray emission; often used for dosimetric and imaging purposes. Therefore many researchers have used ${ }^{188} \mathrm{Re}$ in a variety of fields such as intravascular radiation, radioimmunotherapy using monoclonal antibodies, and metastatic bone lesions (2-4). Radiotherapy using ${ }^{188}$ Re needs novel therapeutic strategies, which can facilitate an increase in the intratumoral uptake or a retention and reduction in its systemic levels. Radiotherapy with intratumorally-injected radiopharmaceuticals is a promising approach to some kinds of tumor such as head and neck cancers because it offers the potential to localize the radiation inside the tumor. Because potential leakage of therapeutic radionuclide causes serious problems in the site of accumulation, there is a need to minimize leakage of the injected compound. Intratumoral injection of ${ }^{188}$ Re HYNIC-PEITf resulted in the highest retention in the tumor mass indicating this approach had strong potential for the treatment of solid tumors.

PEI is a cationic polymer and has been used as a gene delivery vector. Positively charged materials interact with cellular blood components, vascular endothelium, and plasma proteins when they are injected systemically $(18,19)$. In this study, ${ }^{188}$ Re HYNIC-PEI leaked out from injected site showed the liver and lung activity, this might be explained that positive surface charge of PEI led to interactions with lung and hepatic endothelium. Until now, there have been few reports about intratumoral injection using ${ }^{188}$ Re labeled cationic polymer. Therefore, despite of incomplete and preliminary data, our result is thought to be introduced the new, useful compound to the field of radionulide therapy.

High retention of ${ }^{188}$ Re HYNIC-PEI in the tumor demonstrates that the intratumoral injections of positively charged radioactive conjugates bind to tumor cells adjacent to the injection sites. Based on the fact that Tf-PEI derivatives/DNA complexes have been known to deliver the gene efficiently through the Tf-Tf receptor system, in the intratumoral approach of ${ }^{188}$ Re HYNIC-PEI-Tf, Tf-Tf receptor mediated tumoral uptake is thought to play an additional role for higher retention of radiocompound in the tumor than that of ${ }^{188} \mathrm{Re}$ HYNIC-PEI. We certified the existence of Tf-receptor mRNA through RT-PCR method in the Ramos lymphoma cell line. Another role of Tf conjugate as a ligand may be that Tf enlarged the size of ${ }^{188}$ Re labeled polymer because the molecular weight of Tf is relatively heavy, about $80 \mathrm{kDa}$.

As shown in Fig. 1, 2, high retention of ${ }^{188}$ Re labeled cationic polymers in the tumor were not only inspected through nuclear imaging of good quality because of appropriate gamma energy for imaging, but also predicted extensive necrosis around the sites of injection. We verified that injected and retained dose, $11.1 \mathrm{MBq}$, of ${ }^{188} \mathrm{Re}$ HYNIC-PEI-Tf was enough to cause the necrosis to approximately $1 \mathrm{~cm}$-sized tumor through calculated results for dosimetry using the Nodule Module in MIRDOSE3.1 software.

In conclusion, the results of this preliminary study show that ${ }^{188}$ Re labeled HYNIC-PEI-Tf can be a useful radiopharmaceutical agent to treat solid tumors when delivered by intratumoral injection.

\section{ACKNOWLEDGMENT} 2003

This paper was supported by Wonkwang University in

\section{REFERENCES}

1. Knapp FF Jr, Beets AL, Guhlke S, Zamora PO, Bender H, Palmedo $\mathrm{H}$, Biersack HJ. Availability of rhenium-188 from the alumina-based tungsten-188/rhenium-188 generator for preparation of rhenium-188labeled radiopharmaceuticals for cancer treatment. Anticancer Res 1997; 17: 1783-95.

2. Hsieh BT, Hsieh JF, Tsai SC, Lin WY, Huang HT, Ting G, Wang SJ. Rhenium-188-Labeled DTPA: a new radiopharmaceutical for intravascular radiation therapy. Nucl Med Biol 1999; 26: 967-72.

3. Li S, Liu J, Zhang H, Tian M, Wang J, Zheng X. Rhenium-188 HEDP to treat painful bone metastases. Clin Nucl Med 2001; 26: 919-22.

4. Buchmann I, Bunjes D, Kotzerke J, Martin H, Glatting G, Seitz U, Rattat D, Buck A, Dohner H, Reske SN. Myeloablative radioimmunotherapy with Re-188-anti-CD66-antibody for conditioning of highrisk leukemia patients prior to stem cell transplantation: biodistribution, biokinetics and immediate toxicities. Cancer Biother Radiopharm 2002; 17: 151-63.

5. Boussif O, Lezoualc'h F, Zanta MA, Mergny MD, Scherman D, Demeneix B, Behr JP. A versatile vector for gene and oligonucleotide transfer into cells in culture and in vivo: polyethylenimine. Proc Natl Acad Sci USA 1995; 92: 7297-301.

6. Kobayashi S, Hiroishi K, Tokunoh M, Saegusa T. Chelating properties of linear and branched poly (ethylenimines). Macromolecules 1987; 20: 1496-500.

7. Rivas BL, Geckeler KE. Synthesis and metal complexation of poly (ethylenimine) and derivatives. Adv Polym Sci 1992; 102: 173-83.

8. Zanta MA, Boussif O, Adib A, Behr JP. In vitro gene delivery to hepatocytes with galactosylated polyethylenimine. Bioconjug Chem 1997; 8: 839-44.

9. Sagara K, Kim SW. A new synthesis of galactose-poly (ethylene glycol)-polyethylenimine for gene delivery to hepatocytes. J Control Release 2002; 79: 271-81.

10. Kunath K, von Harpe A, Fischer D, Kissel T. Galactose-PEI-DNA complex for targeted gene delivery: degree of substitution affects complex size and transfection efficiency. J Control Release 2003; 88: $159-72$.

11. Ogris M, Steinlein P, Carotta S, Brunner S, Wagner E. DNA/polyethylenimine transfection particles: influence of ligands, polymer size, and PEGylation on internalization and gene expression. AAPS PharmSci 2001; 3: E21.

12. Hildebrandt IJ, Iyer M, Wagner E, Gambhir SS. Optical imaging of transferrin targeted PEI/DNA complexes in living subjects. Gene 
Ther 2003; 10: 758-64.

13. Ogris M, Wagner E. Tumor-targeted gene transfer with DNA polyplexes. Somat Cell Mol Genet 2002; 27: 85-95.

14. Qian ZM, Li H, Sun H, Ho K. Targeted drug delivery via the transferrin receptor-mediated endocytosis pathway. Pharmacol Rev 2002; 54: 561-87.

15. Xu L, Pirollo KF, Chang EH. Transferrin-liposome-mediated p53 sensitization of squamous cell carcinoma of the head and neck to radiation in vitro. Hum Gene Ther 1997; 8: 467-75.

16. Kircheis R, Kichler A, Wallner G, Kursa M, Ogris M, Felzmann T, Buchberger M, Wagner E. Coupling of cell-binding ligands to poly- ethylenimine for targeted gene delivery. Gene Ther 1997; 4: 409-18.

17. Stabin MG. MIRDOSE: personal computer software for internal dose assessment in nuclear medicine. J Nucl Med 1996; 37: 538-46.

18. Ogris M, Brunner S, Schuller S, Kircheis R, Wagner E. PEGylated $D N A /$ transferrin-PEI complexes: reduced interaction with blood components, extended circulation in blood and potential for systemic gene delivery. Gene Ther 1999; 6: 595-605.

19. Oupicky D, Konak C, Dash PR, Seymour LW, Ulbrich K. Effect of albumin and polyanion on the structure of DNA complexes with polycation containing hydrophilic nonionic block. Bioconjug Chem 1999; 10: $764-72$ 